\title{
Entwicklung der \\ Anstellungsbedingungen
}

\section{Thomas Eichenberger}

Dr. iur., Geschäftsleiter VLSS

\author{
Der VLSS ist die Basisorganisation der in der Schweiz tätigen Chefärztinnen und \\ Chefärzte sowie der Leitenden Spitalärztinnen und Spitalärzte innerhalb der FMH. \\ In den Jahren 2003, 2011 und 2016 hat er die Vereinsmitglieder zu ihren Anstel- \\ lungsbedingungen befragt.
}

Die Private Hochschule Wirtschaft PHW (heute ein Teil der Kalaidos Fachhochschule Schweiz, welche u.a. auch einen Executive MBA in Medical Management anbietet) hat im Herbst 2003 im Auftrag der SDK (heute GDK) sowie des VLSS eine umfangreiche Befragung

Der VLSS erhebt nicht den Anspruch, wissenschaftlich fundierte Angaben zur Entwicklung der Einkommen sowie der Anstellungsbedingungen von Kaderärzten in der Schweiz zu machen.

der Schweizer Kaderärztinnen und Kaderärzte sowie bei einigen öffentlichen Spitälern der Schweiz durchgeführt (vgl. SÄZ 2004;85: Nr. 51/52, S. 2754 ff.). Damals konnten 638 von 2838 Fragebogen ausgewertet werden, was aussagekräftige Resultate ergab. Im Jahr 2011 wurde dann lediglich eine stark vereinfachte Gehaltsumfrage auf elektronischem Weg beim gleichen Adressatenkreis durchgeführt, deren Ergebnisse sich nicht für eine Publikation eigneten. Und im Jahr 2016 fand schliesslich eine erneute Umfrage auf elektronischem Weg statt, mit dem Ziel, die Ergebnisse mit früheren Umfragen, insbesondere mit den Ergebnissen des Jahres 2003, vergleichen zu können. Diese Umfrage wurde ausschliesslich unter den Mitgliedern des VLSS durchgeführt. 268 Personen nahmen teil, was einer Rücklaufquote von $25 \%$ entspricht (vgl. VLSS info 12017 : www.vlss.ch).

Der VLSS erhebt nicht den Anspruch, wissenschaftlich fundierte Angaben zur Entwicklung der Einkommen sowie der Anstellungsbedingungen von Kaderärzten in der Schweiz zu machen. Die Umfragen wurden primär für interne Zwecke bzw. für die Zielgruppe der Kaderärztinnen und Kaderärzte durchgeführt. Wir beschränken uns dementsprechend darauf, auf einige unseres Erachtens bedeutsame Trends hinzuweisen. Dabei fliesst selbstverständlich auch die langjährige Erfahrung des Unterzeichnenden im Zusammenhang mit Vertragsberatungen und Gerichtsfällen mit ein.

Weil die drei genannten Umfragen ähnliche Frageraster aufwiesen und auch ähnlich erhoben wurden, konnten wir den folgenden Fragestellungen vertieft nachgehen und dabei auch Entwicklungen über die Zeitachse beleuchten. Ferner nahmen wir die in den Medien thematisierten Ärzte-Boni (vgl. dazu die Sendung ECO des Schweizer Radio und Fernsehen SRF vom 3. Oktober 2016, welche einen Zusammenhang zwischen Ärzte-Boni und Operationszahlen herleiten will) etwas genauer unter die Lupe:

- Sind die Gesamteinkommen der Chefärzte / Leitenden Ärzte gestiegen?

- Haben variable Einkommensbestandteile/Boni zugenommen?

Betrachtet man die Einkommen schweizweit, so zeigt sich zwischen 2002 und 2016 eine stabile Entwicklung.

- Haben sich die Arbeitsbedingungen verändert?

- Wie steht es um die Management-Qualifikationen der Kaderärzte?

- Wie sieht es mit der Berufszufriedenheit aus?

\section{Entwicklung der Gesamteinkommen der Chefärzte / Leitenden Ärzte}

Der VLSS hat die Einkommen der Kaderärzte unabhängig von ihrer medizinischen Fachrichtung erhoben. Betrachtet man die Einkommen schweizweit, so zeigt sich zwischen 2002 und 2016 eine stabile Entwicklung. Insgesamt sind die Gesamteinkommen nominell na- 


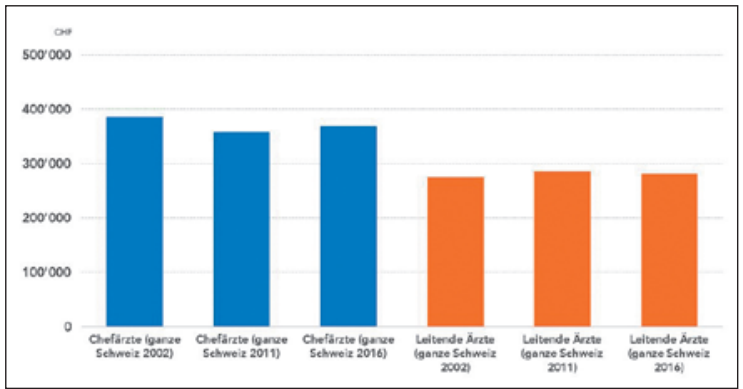

Grafik 1: Einkommensentwicklung Chefärzte und Leitende Ärzte schweizweit.

hezu unverändert geblieben. Dies gilt sowohl für die Chefärzte wie auch für die Leitenden Ärzte. Die Einkommen der Co-Chefärzte sind demgegenüber tendenziell eher rückläufig, wobei sogleich festzuhalten ist, dass wir diesbezüglich nicht über eine ausreichende Datenlage verfügen.

\section{Der VLSS hat sich in Übereinstimmung mit der FMH grundsätzlich ablehnend zu Lohnmodellen geäussert, welche Kaderärzte am Spitalergebnis oder am Ergebnis ihrer Klinik beteiligen.}

Werden die Einkommen der Kaderärzte allerdings nach Regionen unterteilt, zeigen sich unterschiedliche Entwicklungsmuster (siehe Grafik 2). Während sich die Gesamteinkommen in der Region 2 (Kantone FR, GE, JU, NE, TI, VD und VS) im Zeitraum von 2002 bis 2016 anstiegen, gingen sie im gleichen Zeitraum in der Region 3 (GR, AI, AR, GL, SG, SH, TG und ZH) zurück. In den Regionen 1 und 4 dagegen blieben die Einkommen über den gesamten Zeitraum relativ stabil. Alle Regionen bewegten sich aber im Jahr 2016 ungefähr im Bereich eines gesamtschweizerischen Durchschnitts. Selbstverständlich gelten die oberwähnten Vorbehalte, und wir sind nicht in der Lage, die Einkommen nach Fachrichtungen aufzuschlüsseln.

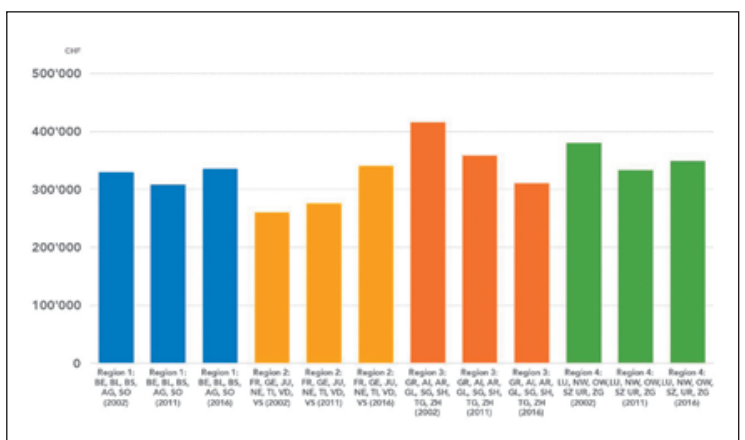

Grafik 2: Einkommensentwicklung der Kaderärzte nach Regionen.
56\% der im Jahr 2016 befragten Mitglieder des VLSS geben denn auch an, dass sich die Einkommensverhältnisse nicht verändert hätten, während $24 \%$ eine Verschlechterung und 20\% eine Verbesserung erfahren haben .

Die durchschnittlichen Einkommen der Schweizer Kaderärzte haben zwischen 2002 und 2016 zwar nicht zugenommen, aber es hat eine regionale Angleichung stattgefunden. Ohne dies werten zu wollen, gehen wir somit davon aus, dass sich die Spitäler auf einem gesamtschweizerischen Markt um Kaderärzte bemühen und dass dieser Markt relativ gut funktioniert.

\section{Bedeutung der Ärzte-Boni}

Die Erhebung zeigt, dass die variablen Einkommensbestandteile (Honorare aus privatärztlicher Tätigkeit oder ergebnisabhängige Boni) seit 2002 von ursprünglich rund 50\% zuerst auf ca. 1/3 zurückgegangen sind und jetzt nur noch bei ca. 10-20\% des Gesamteinkommens liegen. Die Reduktionen bei den variablen Einkommensbestandteilen konnten aber vollumfänglich durch entsprechend höhere Fixeinkommen kompensiert werden. Dies ergibt sich zumindest aus den Durchschnittszahlen, auf welche wir hier abstellen müssen.

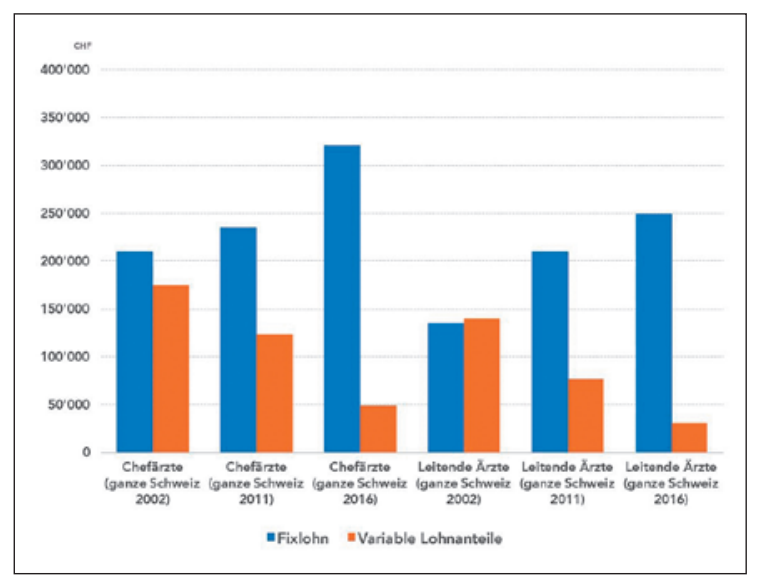

Grafik 3: Veränderung der Lohnkomponenten der Chefärzte und Leitenden Ärzte.

Wir glauben nicht, dass den Kaderärzten an unseren Spitälern bei einem Umfang von 10-20\% des Gesamteinkommens heute noch variable Einkommensbestandteile ausbezahlt werden, welche die Anzahl (unnötiger) Operationen in die Höhe treiben könnte. Der VLSS hat sich aber in Übereinstimmung mit der FMH grundsätzlich ablehnend zu Lohnmodellen geäussert, welche Kaderärzte am Spitalergebnis oder am Ergebnis ihrer Klinik beteiligen (sog. Boni). Er setzt sich stattdessen für die Aufrechterhaltung von Honoraren aus privatärztlicher Tätigkeit ein, weil Privatpatienten und Halbprivatpatienten damit am 


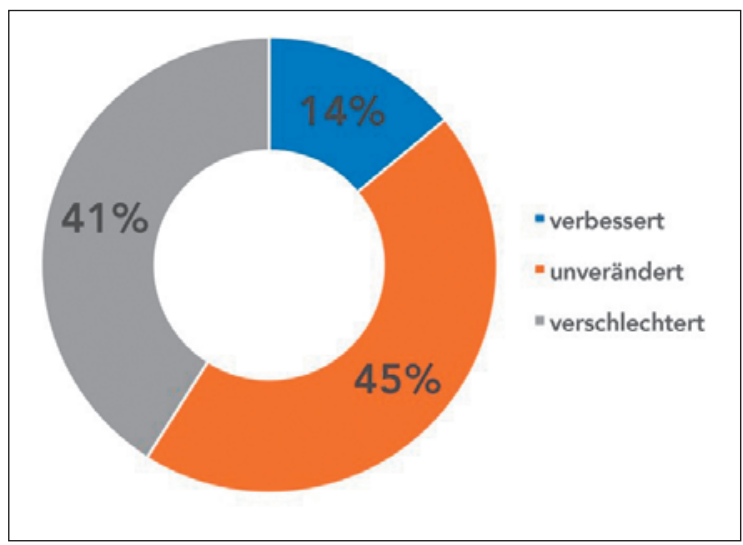

Grafik 4: Veränderungen bei den Arbeitsbedingungen.

Spital in den Genuss einer ganz persönlichen Betreuung durch den von ihnen gewählten Kaderarzt kommen. Dies ist im Gegensatz zur Ausschüttung von Boni ein echter

\section{Erstaunliche $41 \%$ der befragten Kaderärzte geben an, dass sich die Arbeitsbedingungen verschlechtert hätten, während nur $14 \%$ behaupten, die Rahmenbedingungen hätten sich verbessert.}

Mehrwert, denn im Rahmen der Privatpatientenbehandlung findet u.E. keine Entpersonifizierung des ArztPatienten-Verhältnisses und keine Ökonomisierung der Patientenbehandlung statt.

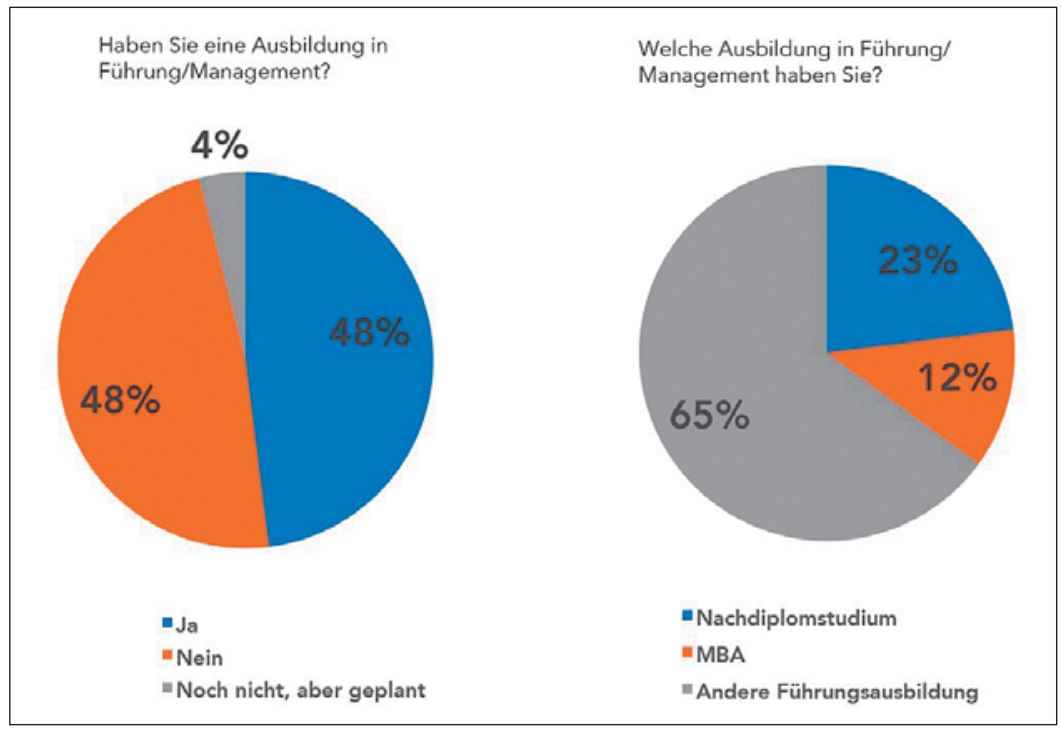

Grafik 5: Ausbildung Führung/Management.

\section{Veränderung der Arbeitsbedingungen}

Erstaunliche $41 \%$ der befragten Kaderärzte geben an, dass sich die Arbeitsbedingungen verschlechtert hätten, während nur $14 \%$ behaupten, die Rahmenbedingungen hätten sich verbessert. Somit beurteilen $45 \%$ der Befragten die Situation als unverändert.

Als Gründe für die Verschlechterung werden die folgenden Umstände zuerst genannt:

- Neue Organisationsstruktur (29\%)

- Mehr Administrationsaufwand (29\%)

- Neue Spitalleitung (19\%)

Einzig die Arbeitszeit hat sich scheinbar verbessert. Während im Jahr 2003 die wöchentliche Gesamtarbeitszeit mit 70 Stunden geschätzt wurde, gaben die befragten Mitglieder des VLSS im Jahr 2016 nur noch 60 Stunden an.

Somit haben sich die Arbeitsbedingungen trotz ungefähr gleich hohem Einkommen im Zeitraum zwischen 2003 und 2016 im Durchschnitt nicht unerheblich verschlechtert. Unsere vielleicht etwas einseitige Analyse, welche zahlreiche Einzelfälle - und damit auch uns bekannte Rechtsstreitigkeiten - mitberücksichtigt, lässt einen engen Zusammenhang zwischen dem Überlebenskampf einzelner Spitäler und den verschlechterten Arbeitsbedingungen vermuten, weil damit oft personelle Veränderungen auf Stufe Verwaltungsrat und Geschäftsleitung und immer wieder neue Organisationsstrukturen einhergehen. Nicht alles, was der Wirtschaft als bewährtes Modell dient, lässt sich auf Spitäler übertragen. Setzen Spitaldirektionen den Hebel zu sehr bei den Kaderärzten an, riskieren sie unweigerlich, langjährig aufgebaute Versorgungsstrukturen zu zerschlagen. Spitäler ohne gute Kaderärzte werden kurz- und mittelfristig verschwinden.

\section{Ausbildung der Kaderärzte in Führung und Management}

48\% der Befragten gaben im Jahr 2016 an, über eine Management-Ausbildung zu verfügen und $4 \%$ haben eine solche geplant. Dies ist sehr erfreulich, hatten doch im Jahr 2003 lediglich 12\% der Befragten angegeben, über eine solche Ausbildung zu verfügen.

Diese Entwicklung zeigt, dass Kaderärzte heute mehrheitlich über das notwendige «Know-how» verfügen, um «gewinnbringend" in der operativen Geschäftsleitung des Spitals Einsitz zu nehmen. Sie sollten dem Verwaltungsrat auch in strategischen Fragen als Ansprechpartner zur Verfügung stehen. Dies ist oft wesentlich günstiger, als bei international tätigen Beratungsfirmen Patentrezepte aus dem Industrie- oder gar Produktionsbereich einzu- 


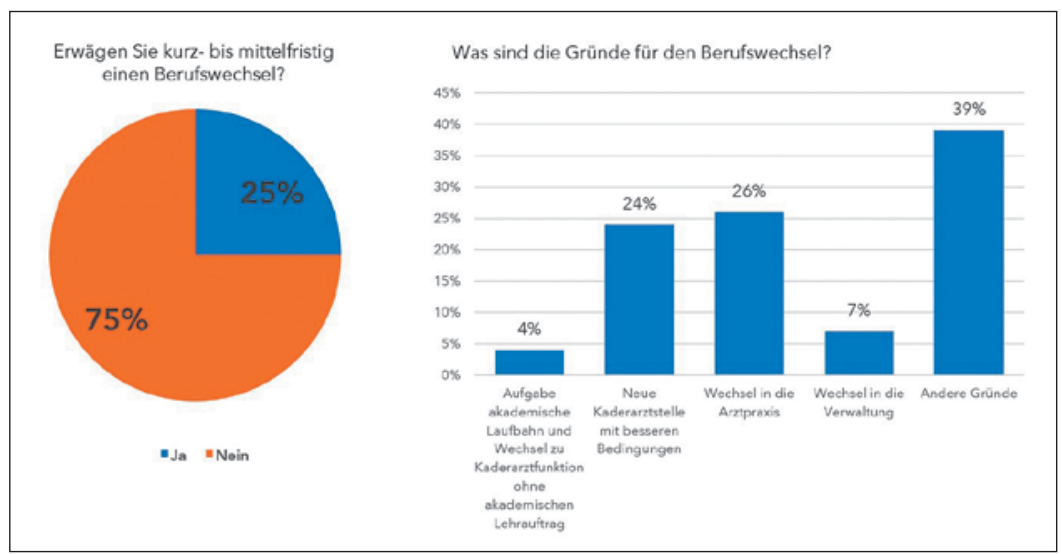

Grafik 6: Berufswechsel und Beweggründe.

\section{Korrespondenz:}

Chefärzte und Leitende

Spitalärzte Schweiz

VLSS

p.A. Postgasse 19

Postfach

CH-3000 Bern 8

info[at]vlss.ch kaufen, welche dann nach einer längeren Phase der versuchten Implementierung am Spital sowieso scheitern, da das Management in einem Spital sehr spezifische Kenntnisse erfordert.

\section{Geplanter Berufswechsel}

Es könnte mit den soeben erwähnten Entwicklungen zusammenhängen, wenn 25\% der befragten VLSS-Mitglieder im Jahr 2016 angaben, einen Berufswechsel zu planen. Nach den Gründen bzw. hinsichtlich der Art des geplanten Berufswechsels gefragt, geben $26 \%$ einen Wechsel in die Arztpraxis und 24\% den Antritt einer Kaderarztstelle mit besseren Bedingungen an. 7\% möchten in die Verwaltung wechseln (sic!) und lediglich $4 \%$ möchten die akademische Laufbahn aufgeben und in eine Kaderarztfunktion ohne akademischen Lehrauftrag wechseln. 39\% der Befragten geben andere Gründe an.

Zusammenfassend zeigt sich ein Handlungsbedarf in Richtung Verbesserung der allgemeinen Arbeitsbedingungen der Kaderärzte. Wenn nicht rechtzeitig dagegen gesteuert wird, droht nach dem Gesagten ein "Braindrain» durch freiwillige Abgänge von teuer ausgebildeten Kaderärztinnen und Kaderärzte in die Arztpraxis, in die Industrie oder in die Verwaltung.

Bildnachweise

(c) VLSS 2017 